\title{
Typicality aids search for an unspecified target, but only in identification and not in attentional guidance
}

\author{
Monica S. Castelhano \\ Queen's University, Kingston, Ontario, Canada \\ AND \\ Alexander Pollatsek and Kyle R. Cave \\ University of Massachusetts, Amherst, Massachusetts
}

\begin{abstract}
Participants searched for a picture of an object, and the object was either a typical or an atypical category member. The object was cued by either the picture or its basic-level category name. Of greatest interest was whether it would be easier to search for typical objects than to search for atypical objects. The answer was "yes," but only in a qualified sense: There was a large typicality effect on response time only for name cues, and almost none of the effect was found in the time to locate (i.e., first fixate) the target. Instead, typicality influenced verification time - the time to respond to the target once it was fixated. Typicality is thus apparently irrelevant when the target is well specified by a picture cue; even when the target is underspecified (as with a name cue), it does not aid attentional guidance, but only facilitates categorization.
\end{abstract}

Many visual search researchers have used simple abstract stimuli, with the target defined by the presence of simple features, such as color and orientation. Real-world searches almost always involve more complex objects, and in many cases the target is less precisely specified. Imagine searching for the phone in a friend's house. You know what phones generally look like but have not seen this particular phone. How do you search for an underspecified target such as this, and how do you know when you have found it? The answer depends on how search targets are represented and how those representations can guide search. What is used as a target representation when only the target's general category, and not its exact appearance, is known? If categorical information can guide search, then objects that are typical of the category should be easier to find than atypical ones. The answer to whether categorical information can guide search is key to understanding real-world searches, but current research offers only limited clues.

Searching is far more efficient with an identical-image cue than with a name cue (Vickery, King, \& Jiang, 2005; Wolfe, Butcher, Lee, \& Hyle, 2003; Wolfe, Horowitz, Kenner, Hyle, \& Vasan, 2004). Wolfe et al. (2004) found that search response times (RTs) were slower with word cues than with picture cues. Moreover, when the picture cue was a different exemplar of the category than the target, performance was no better than with a word cue. However, an exact match between cue and target is not needed. When Vickery et al. cued participants with the exact image of the target, a rotated image, or the target name, performance was better with an image cue than with the name cue, even when the image cue did not depict the exact target orientation.

Why does a lack of the target's visual information in the cue impair search performance? One obvious possibility is that visual information could help guide covert attention and eye movements to the target. For example, in simple conjunctive searches, the target's visual features guide attention. This is true both for monkeys, with higher activation of target features over nontarget features on dimensions such as luminance or color (Motter, 1994; Reynolds, Pasternak, \& Desimone, 2000), and for humans, with saccades tending toward items sharing target features (Houtkamp \& Roelfsema, 2006; Olivers, Meijer, \& Theeuwes, 2006; Scialfa \& Joffe, 1998; Williams \& Reingold, 2001). This guidance is an early component of search, operating before the target is fixated. However, there is another opportunity for visual information about the target to improve search performance: Once an item is fixated, this information may speed the decision about how well the item matches the target template. This later component of search may include time spent subsequently inspecting other items to be sure that they are not the target. Because many visual search studies use conjunctive searches combining a number of simple features, this second step is often not explored. The present study relied on 
an underutilized aspect of eye-movement measures to separate these two components of search. The time until the target is first fixated is a measure of the first component (search guidance); the time between that first fixation and the response is a measure of the second component (target identification).

What kind of template would people use when only given the category name as the search cue? Some type of visual information is probably activated when people are given an object name. For instance, when a category is mentioned, prototypical exemplars are retrieved most easily (Rosch, 1975; Rosch, Mervis, Gray, Johnson, \& Boyes-Braem, 1976). Thus, when a category name defines a search target, a prototypical exemplar might serve as the template for search. If the template contains visual qualities, they may guide eye movements during search, as in conjunctive searches (Scialfa \& Joffe, 1998; Williams \& Reingold, 2001). If so, search should be easier for a named target that is prototypical than for one that is atypical.

In addition to revealing the nature of search-target templates, typicality effects in search guidance would also have implications for a long-standing debate over whether visual search can be guided by categorical information. (See Smilek, Dixon, \& Merikle, 2006, for a review and a recent argument for category effects.) If search can be guided toward items on the basis of their category membership, then this guidance would probably be stronger for typical members than for atypical members, since typicality affects so many aspects of category use.

In the present study, we investigated search guidance by comparing conditions in which a target's picture or its category name was given as a cue. For each target-present condition, the target was either a typical or an atypical exemplar. The stimuli were computer-generated images of real-world objects (see Figure 1). Searches in which the exact visual properties are known were compared with searches for which they are unknown, and searches for targets that presumably closely match expectations for exemplars of a category were compared with searches that match expectations less well.

If typical objects are easier to locate and identify, search for typical objects should be easier, regardless of whether the cue is a picture or the name of the object. On the other hand, typicality may be irrelevant when the cue is a picture, because the picture cue may guide search independently of any derived category information, making typicality relevant only for name cues. When given the category name, participants may generate visual information consistent with a typical category member to help guide the search process. However, the types of featural information
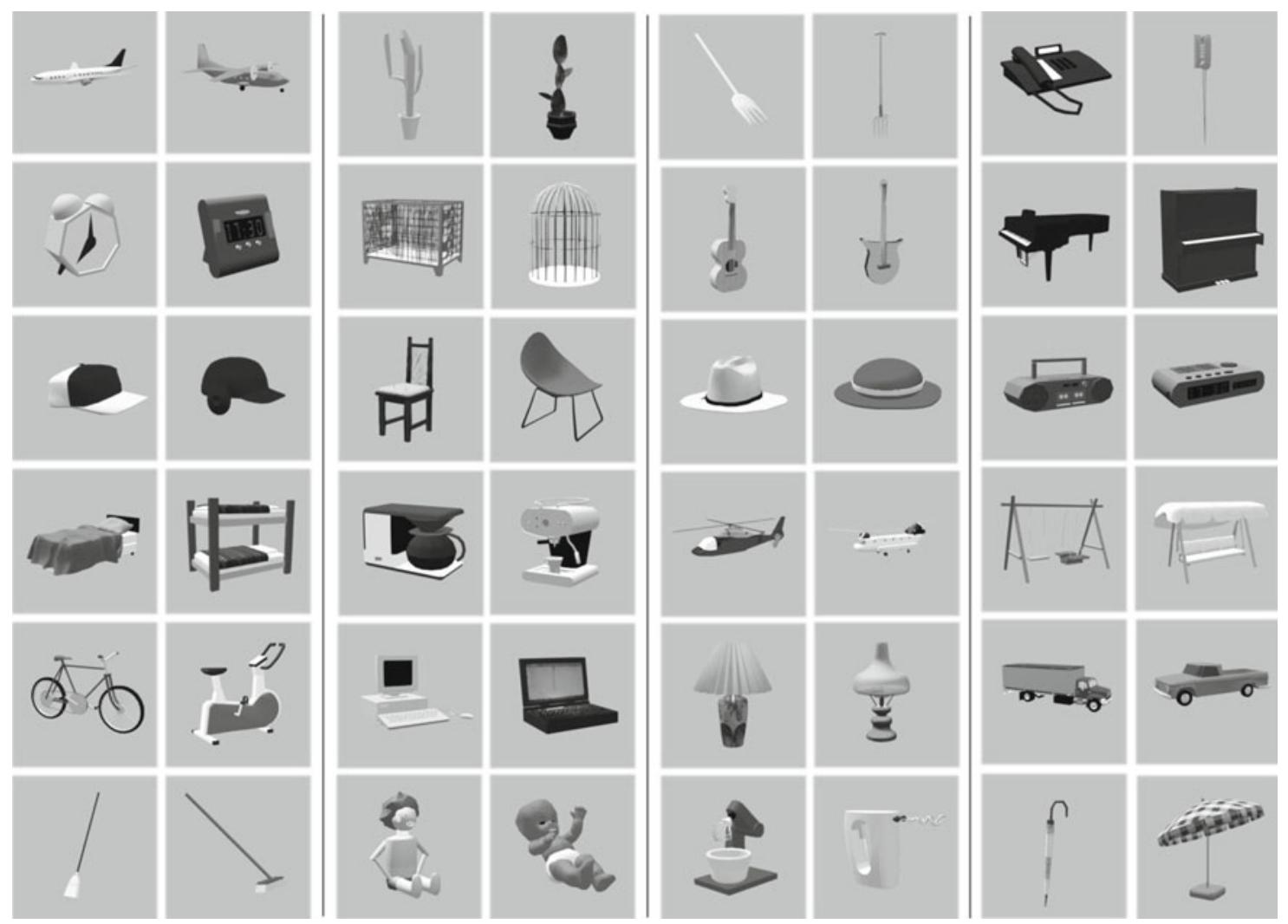

Figure 1. In each of the four pairs of columns, target categories are presented in alphabetical order with typical (left) and atypical (right) exemplars of each. The names of the categories used in the experiment are as follows: Columns 1 and 2, airplane, alarm clock, baseball cap, bed, bicycle, broom; columns 3 and 4, cactus, cage, chair, coffeemaker, computer, doll; columns 5 and 6, fork, guitar, hat, helicopter, lamp, mixer; columns 7 and 8, phone, piano, radio, swing, truck, umbrella). All images were shown in color. 
extracted from such prototypes might be insufficient to guide search effectively. If so, typicality may not help participants locate the target object more quickly. Typicality might still elicit faster responses, however, because visual information generated from a prototype may help identify an object as a category member once attended (i.e., much as it helped identification of single objects in the classic Rosch, 1975, experiments). If so, typicality would affect RTs in the name-cue condition largely by affecting the time to verify the target once it has been fixated.

The eye-movement record was used to divide search time into two components: the target latency (the time from the onset of the search array to the beginning of the first fixation on the target) and the verification time (the time from the beginning of the first fixation on the target to the response). This allows us to determine whether different types of cue information help people to find (i.e., to fixate) the target sooner or merely aid in identifying the target once it is attended.

\section{METHOD}

\section{Participants}

Twelve University of Massachusetts undergraduate students participated for course credit.

\section{Apparatus and Stimuli}

Twenty-four categories were selected, with two targets for each category: a typical and an atypical example (see Figure 1). ${ }^{1}$ In addition, there were 432 distractor items. Objects were computergenerated images individually rendered on Data Becker HomeDesign Studio 5.0 software. Target-present arrays were constructed for each target so that the distractors were similar to the target in color, shape, or both, and there was no semantic coherence between objects in an array. Arrays could contain either 4 or 8 items, arranged in a circular format on a gray background (see Figure 2). Targetabsent arrays were constructed by substituting a nontarget object for the target object. ${ }^{2}$ Target cues presented at the beginning of each trial were either a picture (an image of the target) or a name (the target's basic-level category). A cue mask (hatch symbols arranged in a square) served to mask both the picture and the word cues. There were 96 experimental trials: 4 for each of the 24 categories. The design was counterbalanced: For each category, there were 2 name-cue trials and 2 picture-cue trials, 1 of each in a 4-item and an 8 -item array. Half of the trials were target absent so that no target item was presented more than once.

Eye movements were tracked with an EyeLink II (SR Research) eyetracker. Eye positions were sampled at $500 \mathrm{~Hz}$. Saccades were defined as two or more samples that exceeded a velocity of $30^{\circ} / \mathrm{sec}$; fixations were defined as the times between saccades. The arrays were displayed on an NEC CRT monitor that subtended $33^{\circ} \times 24^{\circ}$ of visual angle and had a refresh rate of $100 \mathrm{~Hz}$. Arrays subtended $19^{\circ}$ across (i.e., from the center of one object to the center of the object directly across from it), and each item in the array was maximally $5.2^{\circ} \times 5.2^{\circ}$ (height and width varied from item to item).

\section{Procedure}

Participants were seated $60 \mathrm{~cm}$ from the monitor, and a chinrest restricted their head movements. After the instructions were presented to the participant, the eyetracker was calibrated. Calibration was assessed before each trial and was recalibrated for each participant if tracking error was greater than $0.4^{\circ}$. At the beginning of each trial, participants fixated a central cross. A target cue appeared, either a picture or a basic-level category name. After $2 \mathrm{sec}$, a visual mask appeared for $500 \mathrm{msec}$ in order to reduce the perception of object motion between the cue and the array. A blank screen was then displayed for $500 \mathrm{msec}$, followed by the search array, which remained until the participants responded. Participants pressed the left button on a button box if the target was present and the right button if it was absent. A target was present on $50 \%$ of the trials. Conditions were counterbalanced across participants, trials were

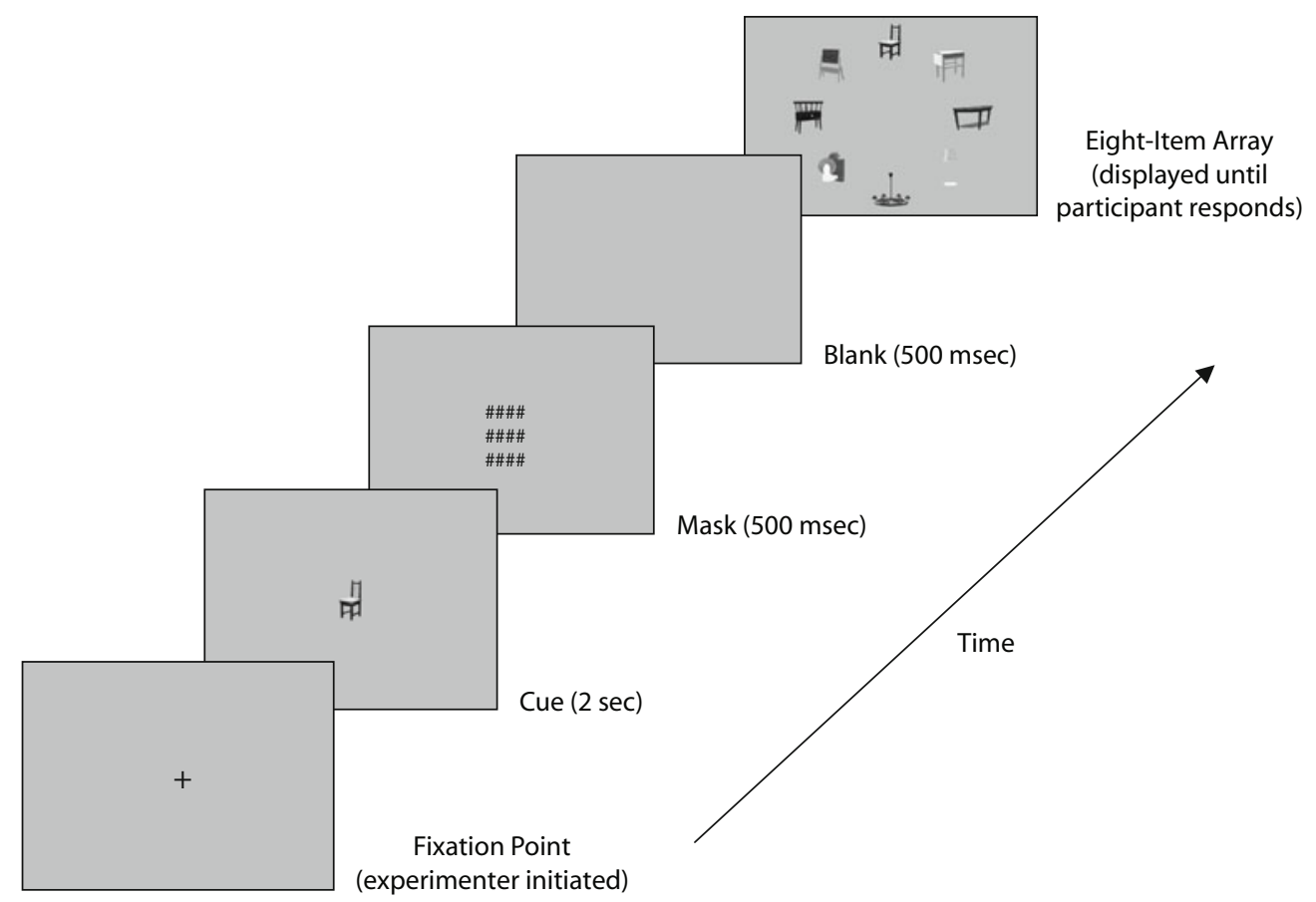

Figure 2. An example trial, in which the participant was given a picture cue of the target (chair was the word cue) and searched through an eight-item array. 
Table 1

Accuracy and Probability of Fixating the Target

\begin{tabular}{|c|c|c|c|c|c|c|c|c|}
\hline & \multicolumn{4}{|c|}{ Name Cue } & \multicolumn{4}{|c|}{ Picture Cue } \\
\hline & \multicolumn{2}{|c|}{ Four-Item Array } & \multicolumn{2}{|c|}{ Eight-Item Array } & \multicolumn{2}{|c|}{ Four-Item Array } & \multicolumn{2}{|c|}{ Eight-Item Array } \\
\hline & Typical & Atypical & Typical & Atypical & Typical & Atypical & Typical & Atypical \\
\hline \multicolumn{9}{|l|}{ Accuracy } \\
\hline Target present & .96 & .78 & .90 & .76 & .99 & .99 & .87 & .97 \\
\hline Target absent & .97 & .96 & .96 & .99 & 1.00 & .99 & 1.00 & .99 \\
\hline Average & .97 & .87 & .93 & .88 & 1.00 & .99 & .94 & .98 \\
\hline Proportion of targets not fixated (correct trials) & .13 & .07 & .05 & .08 & .07 & .17 & .06 & .06 \\
\hline $\begin{array}{l}\text { Proportion of all trials on which the target was } \\
\text { fixated but missed }\end{array}$ & .01 & .22 & .08 & .15 & .01 & .01 & .06 & .03 \\
\hline
\end{tabular}

presented in random order, and no participant saw any array more than once. There were 15 practice trials prior to the 96 experimental trials. Each participant saw the same category four times, but never the same array. (Pilot studies showed that the sheer number of trials prevented participants from being able to keep track of the type of cue and array they had seen before.) None of the objects shown in the practice session were repeated in the experimental session. The entire experiment lasted about $25 \mathrm{~min}$.

\section{RESULTS}

\section{Accuracy}

The overall accuracy was high (94\%), with more misses $(10 \%)$ than false alarms $(2 \%)[F(1,11)=38.44, p<.001$; see Table 1]. Unsurprisingly, there were more errors in searching for a target cued by a name $(9 \%)$ than for a target cued by a picture $(3 \%)[F(1,11)=24.64, p<.001]$, and there were more errors in searching for atypical targets $(7 \%)$ than in searching for typical targets $(4 \%)[F(1,11)=$ $7.16, p<.05]$. However, the effect of set size on error rate was marginal $[F(1,11)=3.3, p=.09]$.

In order to gain further insight into search performance, the sequence of fixations for each trial was also analyzed. For each measure that follows, the typical and atypical targets were compared on the target-present trials. (There was no difference in typicality on target-absent trials.) Table 1 shows the proportion of trials in which a target was correctly reported as present without being fixated. Although targets were less likely to be fixated in four-item than in eight-item arrays $[F(1,11)=7.78, p<.05]$, there was no systematic difference for cue types $(F<1)$ or target types $(F<1)$. However, the proportion of times that a target was fixated but missed (i.e., a target-absent response) was greater for name cues than for picture cues $[F(1,11)=$
26.18, $p<.001]$ and greater for atypical targets than for typical targets $[F(1,11)=17.47, p<.01]$, and there was a significant interaction $[F(1,11)=18.74, p<.001]$. Follow-up analyses indicated that the .14 typicality difference was significant for name cues $[t(11)=-4.98, p<$ $.01]$ but that the .02 typicality difference for picture cues was not $[|t|<1] .^{3}$ This result indicates that, if one is given only a name cue, it is easier to misidentify an atypical target than it is to misidentify a typical target once fixated. The remaining analyses include only correct responses.

\section{RT}

As is shown in Table 2, search was more efficient with picture cues; RTs were $743 \mathrm{msec}$ shorter than they were for name cues $[F(1,11)=76.94, p<.001]$. This cue-type effect was significant for both the typical $[t(11)=3.73$, $p<.01]$ and the atypical $[t(11)=11.30, p<.01]$ targets. RTs were $363 \mathrm{msec}$ shorter for four-item arrays than they were for eight-item arrays $[F(1,11)=38.21, p<.001]$. There was a marginally significant interaction between cue type and set size $[F(1,11)=3.26, p<.09]$, reflecting a larger effect of set size for name cues than for picture cues. More theoretically interesting, there was a significant main effect of typicality $[F(1,11)=5.00, p<.05]$ and a significant typicality $\times$ cue-type interaction $[F(1,11)=5.88$, $p<.05]$, reflecting the fact that the typicality effect was limited to the name-cue condition. The 326-msec typicality effect for the name condition was significant $[t(11)=$ $3.2, p<.01]$, but the $-77-\mathrm{msec}$ effect for the picture-cue condition was not $(|t|<1)$. The interaction of the typicality effect with set size was not significant $(F<1)$.

Thus, when the cue was an image of the target, typicality had no effect on the search time. (If anything, there were

Table 2

Response Times and Eye Movement Measures

\begin{tabular}{|c|c|c|c|c|c|c|c|c|}
\hline \multirow[b]{3}{*}{ Response Measure } & \multicolumn{4}{|c|}{ Name Cue } & \multicolumn{4}{|c|}{ Picture Cue } \\
\hline & \multicolumn{2}{|c|}{ Four-Item Array } & \multicolumn{2}{|c|}{ Eight-Item Array } & \multicolumn{2}{|c|}{ Four-Item Array } & \multicolumn{2}{|c|}{ Eight-Item Array } \\
\hline & Typical & Atypical & Typical & Atypical & Typical & Atypical & Typical & Atypical \\
\hline Response time (msec) & 1,584 & 1,943 & 2,092 & 2,385 & 1,131 & 1,134 & 1,461 & 1,305 \\
\hline Target latency (msec) & 801 & 890 & 1,302 & 1,278 & 755 & 677 & 888 & 813 \\
\hline Fixation count (before target fixated) & 2.50 & 2.61 & 4.30 & 3.94 & 1.38 & 1.45 & 2.06 & 1.96 \\
\hline Target verification $(\mathrm{msec})$ & 679 & 895 & 765 & 1,021 & 428 & 465 & 578 & 514 \\
\hline Total time on target (msec) & 565 & 603 & 531 & 683 & 389 & 429 & 416 & 446 \\
\hline $\begin{array}{l}\text { Total time on distractors (msec; after } \\
\text { target was fixated) }\end{array}$ & 256 & 362 & 328 & 526 & 109 & 97 & 331 & 127 \\
\hline
\end{tabular}


small effects in the opposite direction for both RT and accuracy.) Hence, the physical characteristics of atypical objects did not make them more difficult to locate in search. However, when the cue was only a basic-level category name, the search for the atypical object was more difficult.

\section{Target Latency}

As indicated earlier, typical objects might be more likely to match key features of an image that is generated from a name cue and, thus, might be more likely to attract attention than atypical objects would be. If so, one would expect a typical object to be fixated more quickly than an atypical object. To examine this hypothesis, we examined the target latency: the time from the onset of the search array to the beginning of the first fixation on the target (see Table 2 and Figure 3). It was $290 \mathrm{msec}$ shorter for four-element arrays than for eight-element arrays $[F(1,11)=51.10, p<.001]$ and $285 \mathrm{msec}$ shorter for picture cues than for name cues $[F(1,11)=11.45, p<$ $.01]$. There was also an interaction between cue type and set size $[F(1,11)=8.22, p<.05]$. Further analyses using Tukey's LSD revealed that the difference between set sizes was larger with a name cue $(444 \mathrm{msec})$ than with a picture cue $(135 \mathrm{msec})$. No other interactions were significant. The main effect of typicality on target latency was only $22 \mathrm{msec}(F<1)$. Of greater theoretical interest was that the typicality effect in the name-cue condition was only $32 \mathrm{msec}$ and was not close to significant $(|t|<1)$. Thus, little of the typicality effect on RT can be ascribed to taking less time to locate and fixate a typical target.

\section{Number of Fixations Before Target Fixated}

It is possible, however, that there was greater search efficiency for typical targets but that the latency measure above was not particularly sensitive. Another reasonable measure of search efficiency is the number of fixations made before the target was fixated (excluding the initial central fixation). However, the effects for this measure mirrored those for the latency measure (see Table 2). That is, there were more such fixations with a name cue than with a picture cue $[3.3$ vs. 1.7 fixations; $F(1,11)=46.89$, $p<.01]$, more fixations for the eight-item than for the four-item arrays [3.1 vs. 2.0 fixations; $F(1,11)=39.87$, $p<.01]$, and a significant interaction between cue type and set size $[F(1,11)=10.00, p<.01]$, indicating that the difference between cue types was greater for the eightitem arrays (2.1 fixations) than it was for the four-item arrays (1.1 fixations). However, there was no overall typicality effect on the number of fixations before the target was fixated (typical, 2.6 fixations; atypical, 2.5 fixations; $F<1$ ), nor any typicality effect in the name-cue condition (typical, 3.4; atypical, 3.3; $F<1$ ). No other effects or interactions were significant.

\section{Target Verification}

Verification time, the time from the beginning of the first fixation on the target until the response, was much shorter for picture cues $(496 \mathrm{msec})$ than for name cues $(840 \mathrm{msec})[F(1,11)=69.42, p<.01]$ (see Table 2 and Figure 3). More important, there was a main effect of typicality $[F(1,11)=22.43, p<.01]$ and a significant interaction of typicality and cue type $[F(1,11)=14.25$, $p<.01]$, which reflected the fact that the typicality effect was restricted to the name-cue condition. The 236-msec typicality effect in the name-cue condition was significant $[t(11)=-5.13, p<.01]$, but the -14 -msec effect in the picture-cue condition was not $(|t|<1)$. The 103 -msec difference between the eight-item and the four-item arrays was also significant $[F(1,11)=6.61, p<.05]$. No other interactions were significant. Figure 3 illustrates how la-

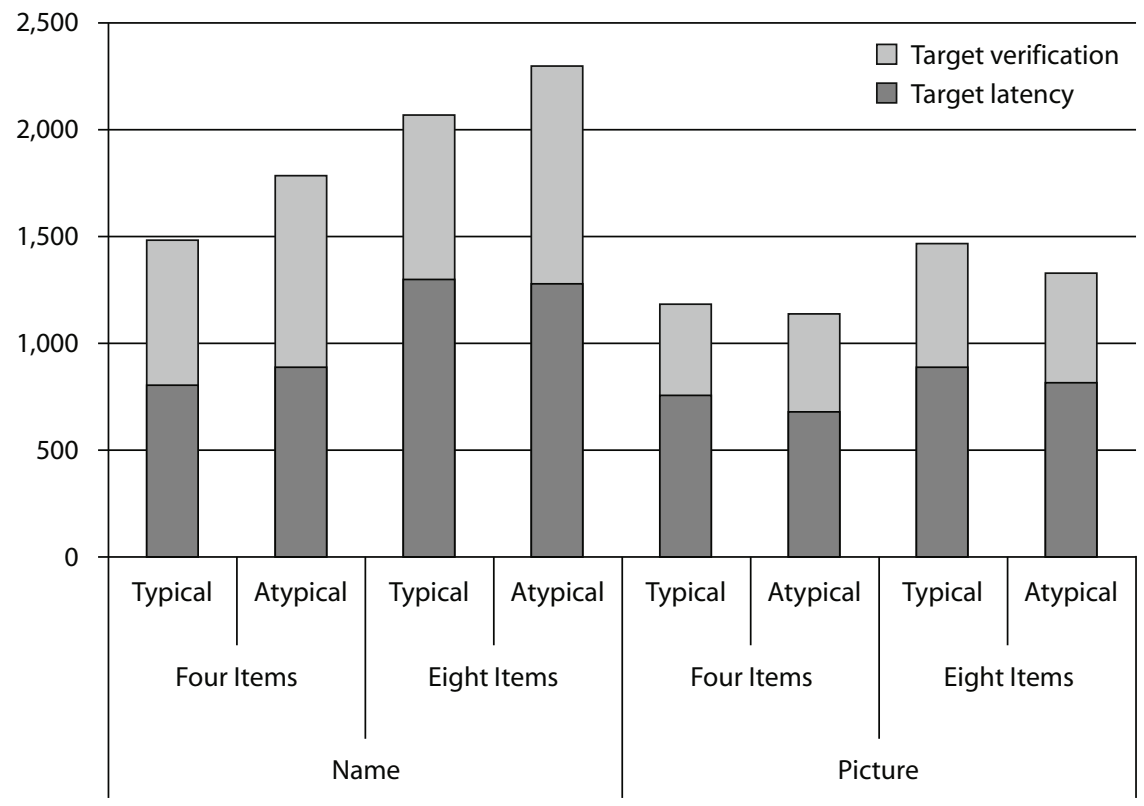

Figure 3. Target latency and target verification times (in milliseconds) for each experimental condition. 
tency was strongly affected only by set size and cue type, whereas verification time was affected by typicality, but only in the name-cue condition.

\section{Where Are the Eyes During Verification?}

One obvious question that arises from the targetverification measure is how much of that time is spent fixating the target, as opposed to fixating distractors. The mean duration of all fixations on the target were summed for each condition (see Table 2). Name-cue search targets were fixated longer $(596 \mathrm{msec})$ than picture-cue targets $(420 \mathrm{msec})$ $[F(1,11)=18.93, p<.01]$. More important, total fixation time for atypical objects $(540 \mathrm{msec})$ was greater than that for typical objects $(475 \mathrm{msec})[F(1,11)=5.8, p<.05]$. Although neither the interaction between typicality and cue condition $(F<1)$ nor the direct comparison of typical and atypical objects when a picture cue was shown $(|t|<1)$ was significant, the 95-msec effect of typicality in the name-cue condition was marginally significant $[t(11)=-1.82, p=$ .09]. No other effects or interactions were significant.

We also analyzed the remaining part of target verification: the total time on all distractors after the target was initially fixated. ${ }^{4}$ There was a main effect of cue type: The double-checking time on distractors was $368 \mathrm{msec}$ for name cues but only $166 \mathrm{msec}$ for picture cues $[F(1,11)=$ $10.74, p<.01]$. There was an effect of array size: Distractor time was $328 \mathrm{msec}$ for eight-item arrays and $206 \mathrm{msec}$ for four-item arrays $[F(1,11)=8.05, p<.05$; see Table 2]. Overall there was only a 22 -msec difference between typical $(256 \mathrm{msec})$ and atypical $(278 \mathrm{msec})$ targets $(F<1)$, but there was a significant interaction of typicality with cue type $[F(1,11)=7.14, p<.05]$. For the name-cue condition, there was a 152 -msec typicality effect $[t(11)=$ $2.54, p<.05]$, but for the picture-cue condition there was a 108-msec "antitypicality effect" that was marginally significant $[t(11)=-1.81, p=.097]$.

\section{DISCUSSION}

In the present study, we explored how searches in which the target was specified only with its basic-level category name differed from searches in which a picture cue was shown and how searches differed for items that were typical or atypical of a basic-level semantic category. The behavioral results were similar to those in previous studies; participants made more errors and were slower in responding when the target was cued by a name rather than by an image. In the present study, we also expanded on these previous studies by showing a large typicality effect (over $300 \mathrm{msec}$ ) in the name-cue condition but no typicality effect in the picture-cue condition. We also revealed the stage of search affected by these factors, using the latency to fixate the target and the verification time once the target was fixated.

The comparison of picture cues with name cues indicated both faster latency times and faster verification times for picture cues. This was true for both typical and atypical targets (see Figure 3). Thus, seeing an image of the target provided cues that both guided attention to the target and speeded identification once it had been fixated. Further- more, the typicality of the target affected performance only with name cues, indicating that atypical objects were not intrinsically harder to process as visual images than were typical objects. Of greatest interest, perhaps, is that the benefit for typical targets in the name-cue condition was not due to better guidance to the target. Instead, virtually all of the benefit was in the time it took to verify that the fixated object was the target. This verification difficulty for atypical targets was mirrored in the error data: In the name-cue condition for trials in which the target was fixated, there were $14 \%$ more errors for atypical targets than for typical targets. The fact that the typicality effect in the name-cue condition was the same for four-item and eightitem arrays also indicates that little of the benefit for typical targets was in initially locating the target.

The lack of a reliable typicality effect in search guidance raises doubts about the guidance of visual search by categorical information. Virtually all of the effect of typicality on the search efficiencies in the present experiment arose from the time to verify the target. These results offer no support for guidance by category and are more consistent with search that is guided only by physical properties, such as color and shape. Any effects of category observed on search times, therefore, could be a result of the time taken to verify the target, not of the time in the search itself. It is, of course, possible that typicality could guide search in certain real-world circumstances, such as when typical items have a certain distinctive visual feature that is not shared either by atypical category members or by the distractors. However, in the circumstances tested here, categorical information (indexed by typicality measures) has at best minimal effects on the initial search before the target is fixated, but nonetheless strongly influences the verification of the target's category.

Going back to our earlier example, when looking for your friend's phone, if it is rather long and sleek, you will probably find it quickly. However, if he or she owns a Mickey Mouse phone with mouse ears, chances are that once you fixate it, you will take some time to realize that it is the phone you are looking for-if you realize it at all.

\section{AUTHOR NOTE}

This study was supported by a grant from the Microsoft Corporation to Keith Rayner. Correspondence concerning this article should be addressed to M. S. Castelhano, Department of Psychology, Queen's University, Kingston, ON, K7L 3N6 Canada (e-mail: monica.castelhano@ queensu.ca)

\section{REFERENCES}

HoutKamp, R., \& Roelfsema, P. R. (2006). The effect of items in working memory on the deployment of attention and the eyes during visual search. Journal of Experimental Psychology: Human Perception \& Performance, 32, 423-442.

Motter, B. C. (1994). Neural correlates of feature selective memory and pop-out in extrastriate area V4. Journal of Neuroscience, 14, 2190-2199.

Olivers, C. N. L., Meiser, F., \& Theeuwes, J. (2006). Feature-based memory-driven attentional capture: Visual working memory content affects visual attention. Journal of Experimental Psychology: Human Perception \& Performance, 32, 1243-1265.

Reynolds, J. H., Pasternak, T., \& Desimone, R. (2000). Attention increases sensitivity of V4 neurons. Neuron, 26, 703-714. 
Rosch, E. [H.] (1975). Cognitive representations of semantic categories. Journal of Experimental Psychology: General, 104, 192-233.

Rosch, E. H., Mervis, C. B., Gray, W. D., Johnson, D. M., \& BoyesBraem, P. (1976). Basic objects in natural categories. Cognitive Psychology, 8, 382-439.

Scialfa, C. T., \& Joffe, K. M. (1998). Response times and eye movements in feature and conjunction search as a function of target eccentricity. Perception \& Psychophysics, 60, 1067-1082.

Smilek, D., Dixon, M. J., \& Merikle, P. M. (2006). Revisiting the category effect: The influence of meaning and search strategy on the efficiency of visual search. Brain Research, 1080, 73-90.

VickeRY, T. J., KING, L.-W., \& JiAng, Y. (2005). Setting up the target template in visual search. Journal of Vision, 5, 81-92.

Williams, D. E., \& Reingold, E. M. (2001). Preattentive guidance of eye movements during triple conjunction search tasks: The effects of feature discriminability and saccadic amplitude. Psychonomic Bulletin \& Review, 8, 476-488

Wolfe, J. M., Butcher, S. J., Lee, C., \& Hyle, M. (2003). Changing your mind: On the contributions of top-down and bottom-up guidance in visual search for feature singletons. Journal of Experimental Psychology: Human Perception \& Performance, 29, 483-502.

Wolfe, J. M., Horowitz, T. S., Kenner, N., Hyle, M., \& Vasan, N. (2004). How fast can you change your mind? The speed of top-down guidance in visual search. Vision Research, 44, 1411-1426.

\section{NOTES}

1. Typical and atypical objects were rated by a separate group of participants $(n=50)$, who saw one of the two category members displayed individually and rated how typical the object was for the named category $(1$, typical, to 7 , not at all typical). The mean rating was 2.2 $(S D=1.9)$ for typical objects and $3.9(S D=2.0)$ for atypical objects $[t(9)=-6.97, p<.01]$. Reliability measures were also calculated for the typical and atypical objects, resulting in alphas of .83 and .80 , respectively.

2. The 48 distractors used in place of the targets in nontarget arrays were not used as distractors in other arrays.

3. However, the small difference in the picture-cue condition could be due to a floor effect.

4. It is not strictly true that verification time is the sum of the total time on the target object and the total time on all distractors (after the target was fixated), since participants would occasionally fixate on something other than one of the objects (e.g., the center of the screen).

(Manuscript received September 18, 2007; revision accepted for publication February 27, 2008.) 